\title{
Neonatal intestinal obstruction secondary to mid-gut volvulus complicated by bowel gangrene in a neonate with ileal atresia: a case report
}

\author{
Baba Usman Ahmadu ${ }^{1, *}$, Yakubu Mava ${ }^{2}$, Joshua Habila Sharah ${ }^{3}$, Abdurrahman Raji Maryam ${ }^{3}$, \\ Paul Dogra ${ }^{3}$, Salihu Akbar ${ }^{3}$ \\ ${ }^{1}$ Department of Paediatrics, Federal Medical Centre Yola, P. O. Box 200, Jimeta-Yola, Adamawa State, Nigeria \\ ${ }^{2}$ Department of Paediatrics Bingham University, Jos, Plateau state, Nigeria; Visiting Consultant Paediatrician FMC Yola \\ ${ }^{3}$ Department of Paediatrics Federal Medical Centre Yola, Nigeria
}

\section{Email address:}

ahmadu4u2003@yahoo.com(B. U. Ahmadu)

\section{To cite this article:}

Baba Usman Ahmadu, Yakubu Mava, Joshua Habila Sharah, Abdurrahman Raji Maryam, Paul Dogra, Salihu Akbar. Neonatal Intestinal Obstruction Secondary to Mid-Gut Volvulus Complicated by Bowel Gangrene in a Neonate with Ileal Atresia: A Case Report. Clinical Medicine Research. Vol. 2, No. 5, 2013, pp. 101-104. doi: 10.11648/j.cmr.20130205.12

\begin{abstract}
Mid-gut volvulus in the absence of malrotation of the gut is rare in neonates and rarer in term babies and can be fatal if intervention is delayed. Herein we report a case of a neonate who had billous vomiting, abdominal distension and moderate dehydration. His abdominal radiograph showed gaseous distension of the stomach, ground glass pattern and calcifications. Exploratory laparotomy revealed mid-gut volvulus complicated by atresia and 75 centimeter length of intestinal gangrene involving the distal one-third of the jejunum and ileum. However, no evidence of malrotation of the gut was found. Resection of the gangrenous segment with jejuno-caecal anastomosis was carried out in addition to blood transfusion, antibiotic and fluid therapy. Patient's condition improved, he passed stools on the 5th postoperative day, and oral sips were commenced.
\end{abstract}

Keywords: Mid-Gut Volvulus without Malrotation, Term Neonate, Special Care Baby Unit, Federal Medical Centre Yola, Nigeria

\section{Introduction}

Mid-gut volvulus without malrotation causing intestinal obstruction in neonates is not a common finding. (1) The incidence of neonatal intestinal obstruction in general is estimated to be 1 in 5,000 live births, however, the incidence rate for developing countries especially Africa are unknown. (2) Reason being that many infants with neonatal intestinal obstruction die without being diagnosed in the hospital. (2) Neonates suspected with midgut volvulus and atresias are described as being sick, having a distended abdomen, and bile-stained vomittus. (3) In 1955, Louw and Barnard, (4) from Cape Town, South Africa reported that volvulus was associated with intestinal atresia as a result of kinking, stretching and disruption of blood flow to the foetal gut. In Nigeria, Ameh and Nmadu, (5) in 2000 have reported that congenital bands, duplication cyst, meconium plug, meckel's diverticulum and internal hernias are some of the causes of secondary mid-gut volvulus.
While on the other hand, Raghu et al, (1) in 2003 in India have published the existence of a primary mid-gut volvulus, described as bowel loop twisting around its mesenteric axis without a predisposing anomaly. Because the cause of primary segmental volvulus is not known, Usmani et al, (6) and colleagues (1) have suggested increased intraabdominal pressure and hyper-motility of the gut as possible causes.

Prompt diagnosis of mid-gut volvulus is important to prevent the potentially fatal complication of intestinal necrosis. (3) In this regard, Gilbertson-Dahdal et al, (3) in Arizona in 2009 found the double bubble sign to be an important guide suggesting duodenal atresia as well as other causes of duodenal obstruction such as midgut volvulus. We present a case of neonatal intestinal obstruction secondary to mid-gut volvulus complicated by bowel gangrene in a neonate with ileal atresia from the Special Care Baby Unit of the Federal Medical Center Yola, Nigeria. 


\section{Case Summary}

A 4 day old neonate delivered at term had bilious vomittus and abdominal distension. Physical examination showed moderate dehydration and a soft ovoid distended abdomen with peritoneal signs. Nasogastric aspirate yielded about $10 \mathrm{mls}$ of billous fluids. Abdominal radiograph showed gaseous distension of the stomach, ground glass pattern and calcified areas (Fig. 1). Lack of relevant facility hindered us from doing an upper gastrointestinal (GI) series. Surgical finding showed midgut volvulus complicated by atresia and 75 centimeter length of intestinal gangrene involving the distal one-third of the jejunum and ileum (Fig. 2). There was no evidence of malrotation. Resection of the gangrenous segment with jejunocaecal anastomosis was carried out in addition to blood transfusion, antibiotic and fluid therapy. The neonates' condition improved after surgery, he passed stools on the 5th postoperative day, and oral sips were commenced.

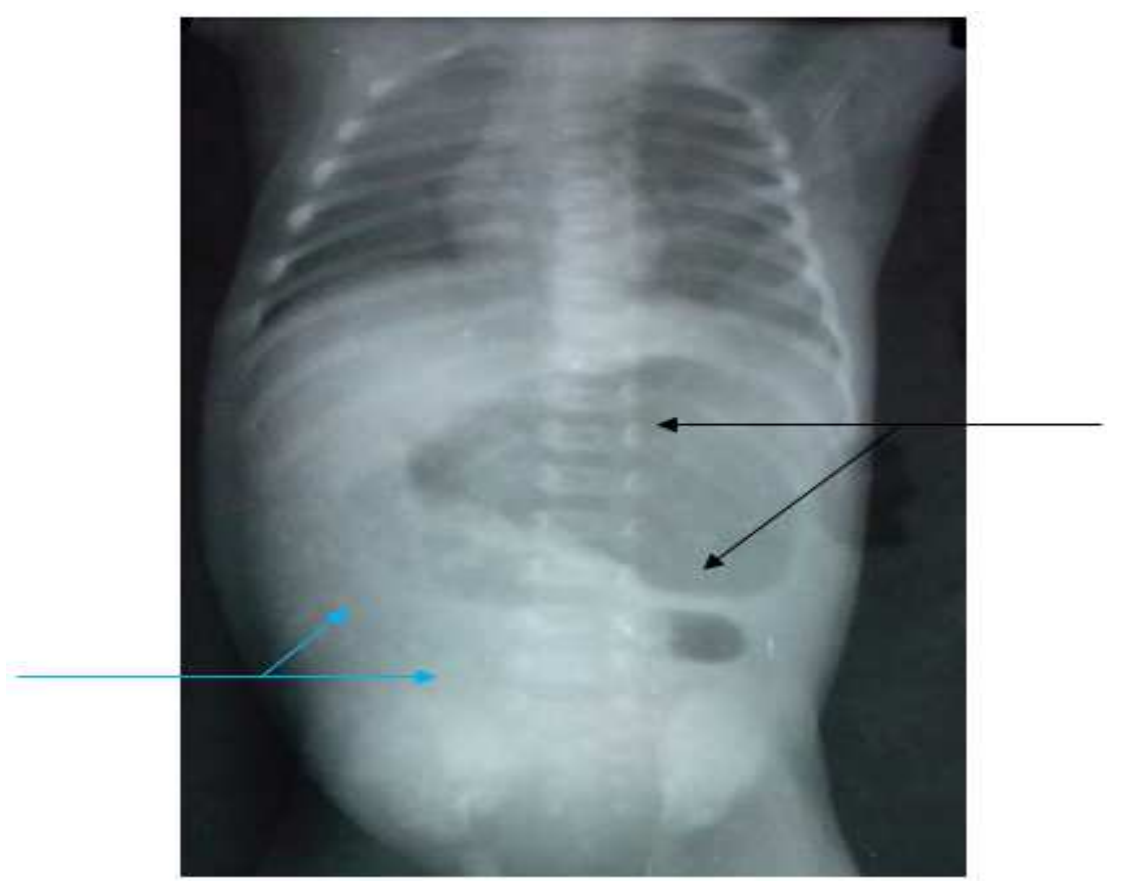

Fig. 1. Abdominal radiograph (Black arrow points to distended stomach while blue arrow shows ground glass pattern and calcification)

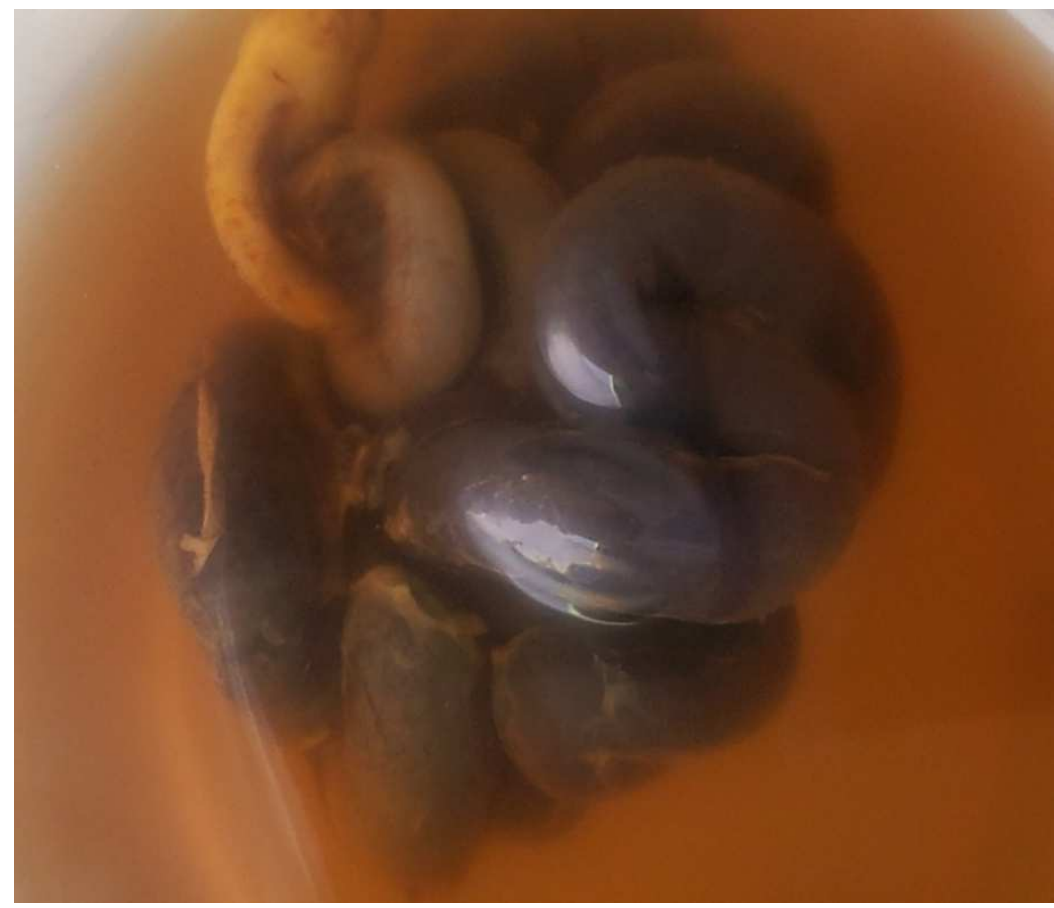

Fig. 2. Image showing resected gangrenous and atretic part of the intestine 


\section{Discussions}

Neonatal intestinal obstruction in general is one of the most common emergency situations that occur during the neonatal period. (2) However, mid-gut volvulus without malrotation of the gut as a cause of neonatal intestinal obstruction is a rare finding. (1-3) In fact, many authors have reported that mid-gut volvulus without malrotation of the gut occurs in less than $30 \%$ of all cases. (7) Mid-gut volvulus was observed without evidence of malrotation of the gut in this infant. Unlike other studies that had found secondary causes of volvulus, no particular cause was identified herein; meaning that the index case belonged to a rare type called primary segmental volvulus. (1-3) The ileal atresia observed in current case could have originated from inadequate blood supply to the ileum arising from the midgut torsion. Jung et al, (8) in 2011 found that primary segmental volvulus although rare is mostly seen in preterm babies and rarely in term babies. Preterm babies have a relatively small sized abdomen that may not accommodate all gut content without a greater rise in intra-abdominal pressure when compared to term babies. This could in part explain the higher occurrence of volvulus without malrotation in preterm babies. The current case was observed in a term baby, which has been reported previously. (1)

Cases of mid-gut volvulus with neonatal intestinal obstruction are characterized by histories of polyhydramnious, bile stained vomittus and abdominal distension. (1-3, 6-8) All these were observed in the current case. Evidence of aspiration pneumonia, malnutrition, bowel perforation, calcifications and dehydration could be found on examination especially if the patient presented late to the hospital. (2) Calcifications and moderate dehydration that was promptly managed were found on this patient.

Abdominal X-ray is a very useful investigation that may scale down the number of possible causes of neonatal intestinal obstruction. Double bubble sign characterized by two air-filled regions corresponding to a distended stomach and proximal duodenum could suggest atresias, and volvulus e.t.c. $(3,9)$ However, its absence as was observed in the current case does not rule out the aforesaid possibilities. This is because our patient was on nasogastric tube (NGT) for drainage of stomach contents. Passing a NGT tube for decompression and drainage of gastric contents could collapse the duodenal bulb thereby making this sign negative. $(3,9)$ In such cases, repeat abdominal Xray after injection of air contrast has been advocated. However, this was not performed on the present case in order to minimize the patient's exposure to radiation. Barium studies may also be useful in managing cases of intestinal obstruction secondary to atresias, volvulus e.t.c, however, lack of facility hindered us from doing it.

Bowel gangrene as well as ileal atresia were found on the patient at exploratory laparotomy. These were similar to the findings by Kitano et al, (7) in 1995. The colon usually gives a cushion effect to the small intestines; this mitigating effect may be lacking in cases of mid-gut volvulus associated with malrotation of the gut. (1) The result of this is rapid development of bowel gangrene as was seen in the recent case. Vergnes et al, (10) in 1989, however, argued that gangrene in cases of mid-gut volvulus associated with gut malrotation was due to a fixed caecum giving rise to a tighter volvulus leading to rapid ischemia and irreversible necrosis.

\section{Conclusion}

Resection of the twisted gangrenous and atretic bowel is necessary in neonates diagnosed with this rare condition, hence the need for early and prompt intervention in order to improve neonatal survival.

\section{Authors' Contributions}

BUA accessed and wrote the initial draft of the manuscript. All authors were involved in managing the patient, analysis of the images and approval of the final manuscript.

\section{Conflict of Interest: Nil}

\section{Funding Support: None}

\section{References}

[1] Raghu S, Sadashiva R, Kishan BS. Primary Segmental Volvulus Mimicking Ileal Atresia. J Neonat Surg 2013;2:69.

[2] Ekenze SO, Ibeziako SN, Ezomike UO. Trends in neonatal intestinal obstruction in a developing country, 1996-2005. World J Surg 2007;31:2405-09.

[3] Gilbertson-Dahdal DL, Dutta S, Varich LJ, Barth RA. Neonatal Malrotation with Midgut Volvulus Mimicking Duodenal Atresia. AJR 2009; 192:1269-71.

[4] Louw JH, Barnard CN. Congenital intestinal atresia: observations on its origin. Lancet 1955;269:1065-67.

[5] Ameh EA, Nmadu PT. Intestinal volvulus: aetiology, morbidity, and mortality in Nigerian children. Pediatr Surg Int 2000;16:50-2.

[6] Usmani SS, Kenigsberg K. Intrauterine volvulus without malrotation. J Pediatr Surg 1991;26:1409-10.

[7] Kitano Y, Hashizume K, Okhura M. Segmental small bowel volvulus not associated with malrotation in childhood. Pediatr Surg Int 1995;10:335-38.

[8] Jung E, Choi SO, Park WH. Primary segmental volvulus of the ileum mimicking meconium plug syndrome. J Korean Surg Soc. 2011;80: 85-7. 
[9] Traubici J. The double bubble sign. Radiol 2001;220:46364.
[10] Vergnes Boissinot F, Pontailler JR. Primary volvulus of the small intestine without malrotation. Apropos of 7 cases. Ann Pediatr 1989;36:141-7. 